\title{
DINÁMICAS DEL CONOCIMIENTO CIENTÍFICO DE LA FACULTAD DE CIENCIAS EXACTAS Y NATURALES Y AGRIMENSURA DE LA UNNE
}

Ing. Miguel Angel Ibañez ${ }^{1}, \mathrm{Mg}$. Ing. Rubén Edgar Corvalán², Ing Norberto Argentino Sanabria ${ }^{3}$

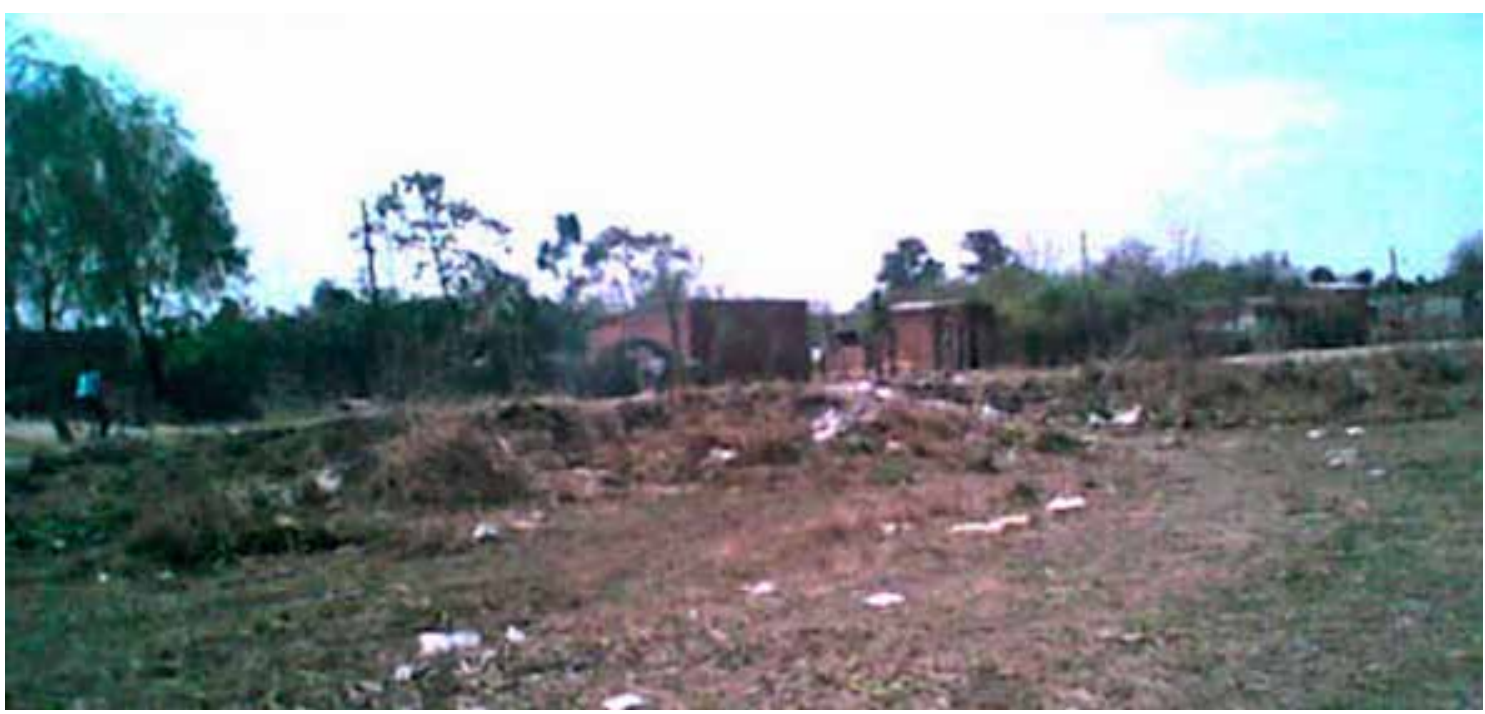

\section{RESUMEN:}

Para poder evaluar el grado de conocimiento de la población de los barrios periféricos de la ciudad de Corrientes, sobre las actividades que realiza la facultad, y su relación con la nueva tecnología se realizaron algunas preguntas, organizadas en un cuestionario, a personas representativas de la muestra en estudio. Se hacían preguntas sobre ¿qué pensaban de la universidad?, ¿Cómo tomaban los desarrollos tecnológicos creado en dicha entidad?, ¿Cómoles afectaba la tecnología en su vida?, entre otras.
Palabras Clave: Vinculación, difusión, transferencia, conocimiento, universidad, vulnerabilidad

\section{INTRODUCCIÓN:}

Las personas que se entrevistaron, son personas radicadas en los barrios periféricos y ribereños al Río Paraná que se encuentran a varios kilómetros del centro de la Ciudad de Corrientes Capital.

Los barrios entrevistados de la Ciudad de Corriente Capital, fueron: Río Pa-

\footnotetext{
${ }^{1}$ Becario Ingeniero Eléctrico: Miguel Angel Ibañez

2 Director: Mg. Ing. Rubén Edgar Corvalán

${ }^{3}$ Sub Director: Ing Norberto Argentino Sanabria
} 
rana, Barrio "Molina punta", punta Taita1o, Barrio "La Chola", Barrio "La Olla",

Las zonas ribereñas del río Paraná son las ubicadas en: Paso de la Patria y Riachuelo.

La entrevista se hizo a personas vulnerables que tienen fuera de su alcance la mayoría de los recursos que ofrece la sociedad de la Ciudades, las mismas son personas que poseen muy poca formación formal educativa (no terminaron la primar o secundaria) y les cuesta un poco la sociabilización con las personas que residen en las grandes ciudades , como en este caso, Corrientes Capital.

Para este cometido se utilizo un instrumento de recolección de datos (una plani11a). La misma facilito e hizo más dinámica la entrevista y permitió poder analizar de manera práctica los datos obtenidos. La misma es de uso abarcativo para este estudio y es un material utilizado por la practicidad y el orden de la recopilación de datos.

Las dificultades con las que nos encontramos fueron:

El lenguaje que debemos utilizar a la hora de entrevistar, transpolar el lenguaje de la ciencia a la de los entrevistados y el proceso contrario para la decodificación de los resultados.

La aceptación de las personas para ser entrevistadas, que requiere de contactos previos a fin de conseguir un cierto grado de familiaridad que facilite la entrevista.

La disponibilidad de horarios de las personas entrevistadas.

Estas dificultades presentadas, fueron superadas con éxito., gracias a la participación de becarios para la realización de las mismas, bajo la dirección de docentes investigadores con experiencia previa.

\section{MATERIALES, MÉTODOS Y TÉCNICAS APLICADAS:}

La Metodología aplicada y sus adecuaciones con el objeto de estudiar la temática y los objetivos pautados, En función de los referentes conceptuales y los objetivos enunciados, el trabajo se desarrollo fundamentalmente bajo un enfoque descriptivo, involucrando el procesamiento y análisis de información cualitativa y cuantitativa. La información previa provino de varios tipos de fuentes: bibliográficas (las mismas son las nombradas en el proyecto), documentales, estadísticas, de cuestionarios y de entrevistas.

Las técnicas aplicadas fueron de la encuesta, que es un método estructurado que utiliza la comunicación para recolectar información directamente de los integrantes de una muestra mediante la aplicación de un cuestionario, método más conveniente para el desarrollo de las actividades y poder ir avanzando con el pasar del tiempo en forma paulatina, siguiendo los siguientes pasos

1- Identificacion de la población destinataria: población vulnerable de los barrios periféricos de Corrientes, paso de la Patria y Riachuelo

2- Diseño del cuestionario: Instrumento aplicado

3- Diseño muestral: la probabilidad de elección fue la misma para cada individuo, dentro de la población objetivo

4- Acceso y recopilación de la información: de forma oral y escrita

5- Análisis de la información: Se relevaron 57 grupos familiares 


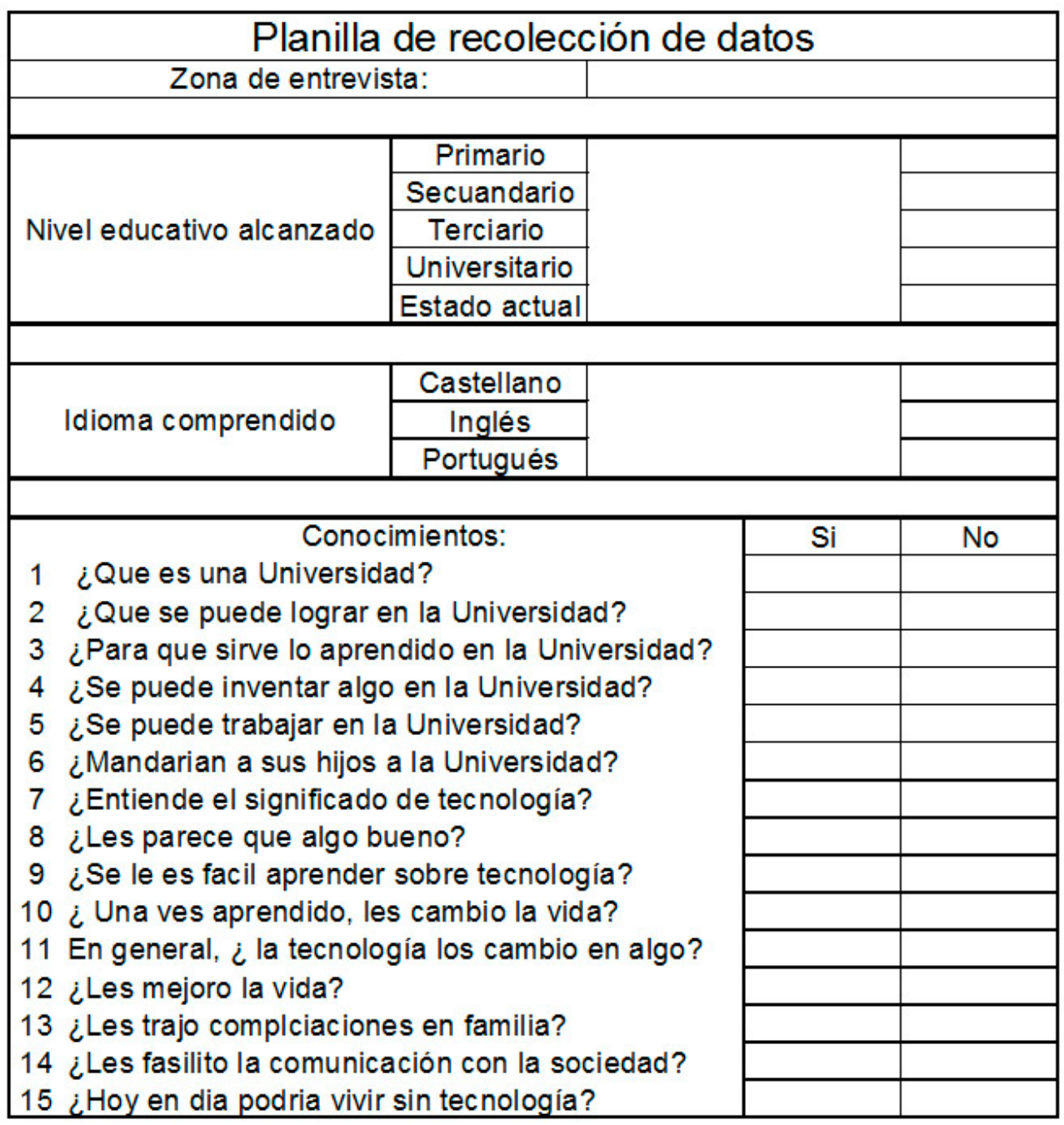

\section{RESULTADOS}

Uno de los resultados obtenidos fue la gran cantidad de personas con desconocimiento de la función investigación (88 \%) de la universidad en general y de la FACENA en particular, oralmente las mismas fueron comentadas, para que se difunda la importancia de estas facetas de la Facultad de Ciencias Exactas y Naturales y Agrimensura y la Universidad Nacional del Nordeste y de lo que eran capaz de realizar los investigadores que se encuentran en la misma.
Otra conjetura confirmada fue que, la tecnología ha cambiado parte de sus vidas $\mathrm{y}$ formas de trabajo (77\%). Esto fue expresado por los individuos de varias maneras, debido a que según ellos la tecnología les ayuda a mejorar su calidad de vida.

Otra hipótesis confirmada es la del grado de interés de las personas jóvenes para seguír sus estudios, en caso de acceder a una beca o su situación lo permitiera (82\%) una vez terminado el secundario. Pero si bien se analiza esta posibilidad. Se ve claramente que no se sienten totalmente incentivados. 


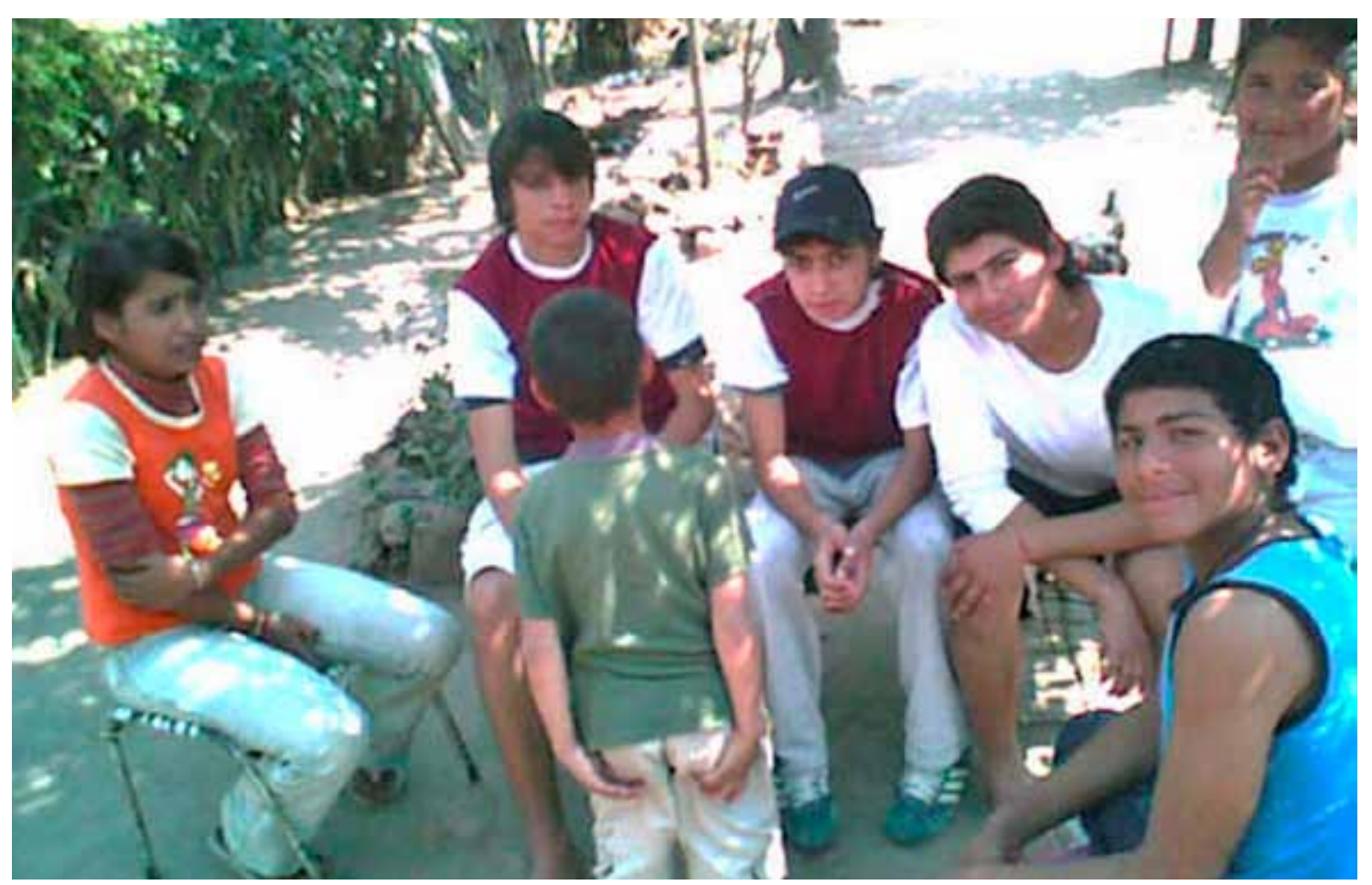

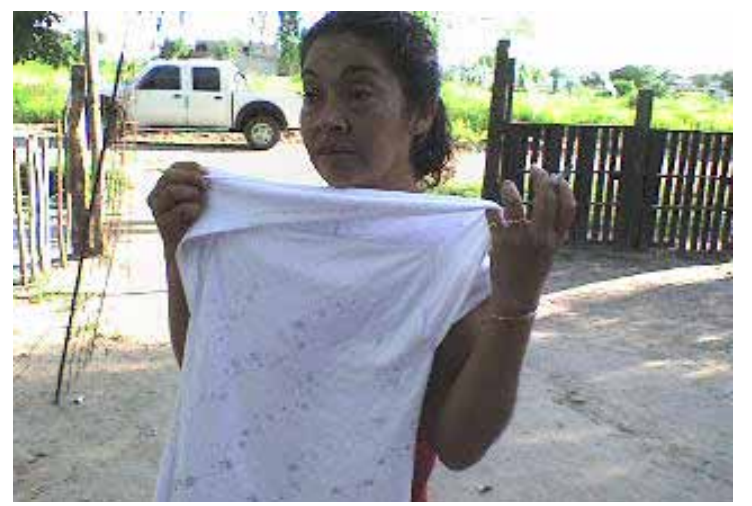

También se pudo confirmar que con la ayuda de la tecnología las personas se encuentran más comunicadas y poseen mayor facilidad a la hora de comunicarse con la sociedad (100\%). Aduciendo que la tecnología es necesaria, es decir, que se les complicaría vivir sin la tecnología actual.

\section{CONCLUSIONES:}

Se llegó a la conclusión que el avance de la tecnología es considerada por las pobla-

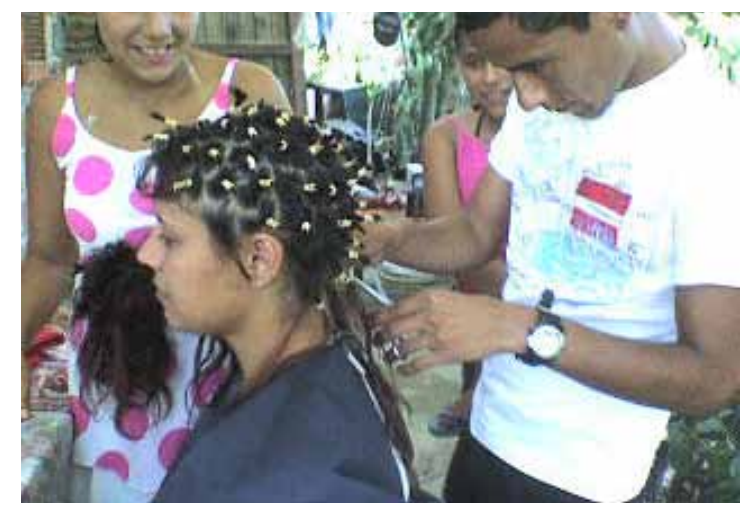

ción estudiada, como un proceso fuera de su vida habitual También se ha constatado la falta de conocimiento sobre la definición y utilización de algunas tecnologías, lo que lleva a disminuir sus posibilidades a la hora de conseguir un puesto laboral mejor remunerado.

Según los resultados de las encuestas, se obtuvo una notoria falta de conocimiento sobre el ámbito universitario y lo que conlleva el mismo en extensión e investigación. 
Esto podría ser producto de falta de información sobre los sistemas educativos. Se destaca la falta de información sobre los proyecto de investigación ejecutados actualmente en la Universidad Nacional del Nordeste.

\section{BIBLIOGRAFÍA:}

Corvalan R.E, Estebanez M.E, Cuevas A.S (2014) Vinculación Científica tecnológica en una universidad argentina. Editorial Académica española.

Estébanez M. E. (2007) Ciencia, tecnología y políticas sociales.. Publicada por Revista Ciencia, Docencia y Tecnología Universidad Nacional De Entre Rios . Paraná, Nro 34, julio 2007.

Guber, Rosana (1990): El salvaje metropolitano: reconstrucción del conocimiento social en el trabajo de campo; Paidós, B.Aires.

Knorr-Cetina, K. (1992): “¿Comunidades científicas o arenas trans-epistémi-
Se constato que la mayoría de la muestra considera que la tecnología ha cambiado su vida, y que les ayuda a vivir mejor debido a que les da un confort diario. Esto hace que se acostumbre a estas comodidades y crea que no pueda llegar a vivir sin estos avances.

cas de investigación? Una crítica de los modelos cuasi-económicos de la ciencia”, en Redes, No 7, Buenos Aires, 1996

Scribano, Adrián (2008): El proceso de investigación social cualitativo; Prometeo, Buenos .Aires

Estébanez, María Elina, García Godoy, Bárbara (2009): Ciencia,tecnologíay desarrollo social. Un enfoque sociológico. Ponencia al Congreso ALAS Buenos Aires, agosto 2009.

Ferro, Silvia (2009) La agricultura familiar en la Argentina: nuevos enfoques para problemas viejos. Chaco: Debates del siglo XXI. Instituto de Cultura de la Provincia de Chaco. 